\title{
Highly Stretchable and Self-Healing Strain Sensors Based on Nanocellulose-Supported Graphene Dispersed in Electro-Conductive Hydrogels
}

\author{
Chunxiao Zheng ${ }^{1}$, Yiying Yue ${ }^{2}, \mathrm{Lu} \mathrm{Gan}^{1}$, Xinwu Xu ${ }^{1}$, Changtong Mei ${ }^{1, *(D)}$ and \\ Jingquan Han $1, *$ (D) \\ 1 College of Materials Science and Engineering, Nanjing Forestry University, Nanjing 210037, China \\ 2 College of Biology and Environment, Nanjing Forestry University, Nanjing 210037, China \\ * Correspondence: mei@njfu.edu.cn (C.M.); hjq@njfu.edu.cn (J.H.)
}

Received: 18 May 2019; Accepted: 11 June 2019; Published: 28 June 2019

check for updates

\begin{abstract}
Intrinsic self-healing and highly stretchable electro-conductive hydrogels demonstrate wide-ranging utilization in intelligent electronic skin. Herein, we propose a new class of strain sensors prepared by cellulose nanofibers (CNFs) and graphene (GN) co-incorporated poly (vinyl alcohol)-borax (GN-CNF@PVA) hydrogel. The borax can reversibly and dynamically associate with poly (vinyl alcohol) (PVA) and GN-CNF nanocomplexes as a cross-linking agent, providing a tough and flexible network with the hydrogels. CNFs act as a bio-template and dispersant to support GN to create homogeneous GN-CNF aqueous dispersion, endowing the GN-CNF@PVA gels with promoted mechanical flexibility, strength and good conductivity. The resulting composite gels have high stretchability (break-up elongation up to 1000\%), excellent viscoelasticity (storage modulus up to $3.7 \mathrm{kPa})$, rapid self-healing ability $(20 \mathrm{~s})$ and high healing efficiency $(97.7 \pm 1.2 \%)$. Due to effective electric pathways provided by GN-CNF nanocomplexes, the strain sensors integrated by GN-CNF@PVA hydrogel with good responsiveness, stability and repeatability can efficiently identify and monitor the various human motions with the gauge factor (GF) of about 3.8, showing promising applications in the field of wearable sensing devices.
\end{abstract}

Keywords: nanocellulose; poly (vinyl alcohol); graphene; borax; hydrogel; self-healing; conductive; stretchable; viscoelasticity; strain sensor

\section{Introduction}

Recently, flexible, stretchable, self-healing and human-friendly devices have gained widespread attention for multi-functional wearable electronics [1,2]. As the essential components in soft electronics, the stretchable strain sensors that can perceive human movement have promoted rapid development of personalized health detection, human motion monitoring, E-skins and robots [3-9]. Hydrogels with a 3D polymer network can preserve large amounts of water. During deformation, these soft materials can maintain their structural integrity and exhibit intrinsic flexibility, stretchability and even self-healing ability, which are considered as a suitable candidate for the fabrication of strain sensors [2]. Currently, electro-conductive hydrogels (ECHs) are emerging as a promising class of functional and intelligent soft materials because of their synergic electro-conductibility, rheological viscoelasticity and inherent flexibility [10]. ECHs generally consist of two parts, a conductive polymer and a hydrogel matrix, and each has its own unique properties. The conductive polymers are embedded in the hydrogel network via copolymerization, grafting or cross-linked reaction, which can retain the excellent properties of the hydrogel while endowing the electron transport capability with hydrogels. However, most ECHs currently suffer from weak mechanical strength, insufficient viscoelasticity, lack of self-healing ability and poor electrical conductivity, which can hardly satisfy the demands of practical applications. 
The introduction of nanocellulose into hydrogel matrixes is an important method to improve their mechanical properties while achieving functionalization. Nanocellulose with cellulose backbone chains inherently forms a hierarchical layered structure. This architecture allows for strong interaction between polymer gel matrix and adjacent nanocellulose, which can contribute to the enhancement of mechanical properties [11-13]. On account of the introduction of -OH groups on polymer chains, poly (vinyl alcohol) (PVA) is capable of forming hydrogels with crosslinking, which exhibit intrinsic hydrophilicity, biocompatibility, biodegradability, high crystallinity and the feasibility of blending with nanocellulose [14]. Because the -OH groups on the nanocellulose surface can construct a stable hydrogen bonding system with the -OH groups of the PVA molecular chains, nanocellulose is ideally suitable for reinforcing nanofillers for PVA hydrogels. Because of their higher aspect ratio, larger size, chain entanglement and the overlap of the elongated nanofibers, cellulose nanofibers (CNFs) can form a more stable and stronger 3D network microstructure over cellulose nanocrystals (CNCs), providing a more significant reinforcing effect on PVA-based composite hydrogels [15].

In order to further endow the hydrogels with electro-conductivity, conductive hydrogels are prepared by introducing conductive materials, including conducting polymers, metallic nanomaterials and carbon nanoparticles [16-19] into hydrogels through a crosslinking approach [20]. Generally, the integration of these conductive materials can also result in the promotion of the toughness and even viscoelasticity of hydrogels due to the nanocomposite-reinforced mechanism [21]. As one of the most promising nanomaterials, graphene (GN) is a monolayer of two-dimensional carbon materials organized laterally in a honeycomb-like lattice [22]. In recent years, people have attempted to increase the mechanical strength and electrical conductivity of composite hydrogels by incorporating conductive nanomaterials. For example, Cai et al. demonstrated a type of CNTs/PVA hybrid hydrogels towards stretchable sensors [23]. Tong et al. found that the introduction of a certain amount of CNTs into the PVA hydrogel could improve the mechanical property and stretchability to some extent [24]. Nevertheless, because GN is very difficult to disperse well in aqueous suspension or hydrogel matrix, the non-homogeneity resulting from GN aggregation has adverse effects on the formation of the gel network, toughness and electro-conductibility for the composite gels, which is still an unsolved issue. Fortunately, CNFs can not only enhance the hydrogel matrix, but also effectively disperse the dispersion of GN in water. Therefore, we believe that the incorporation of nanocellulose can effectively solve this issue.

In addition, self-healing materials have attracted increasing attention. Self-healing hydrogels with reliability, durability and safety are capable of re-establishing their integrity, properties and gel network structure after damage, which can significantly extend the service life of hydrogel-based electronics [11,25]. Self-healing hydrogels are usually achieved by constructing dynamically reversible chemical bonds, such as hydrogen bonds, host-guest interactions and hydrophobic interactions [26,27]. Haick et al. prepared a type of flexible and self-repairing sensing material fabricated via dispersing metal particles in polyurethane diol towards a self-healable electrode [28]. Park et al. developed a class of electroconductive hydrogels with self-healing behavior via a polymerization of pyrrole monomers in agarose solution [25]. Nonetheless, most of the previously reported self-healing electronics suffer from poor stretchability, which can hardly be stretched over $100 \%$. Consequently, it is highly desirable to explore an ECHs platform which can ideally combine self-healing ability, high stretchability and electro-conductibility all together simultaneously.

Herein, we successfully fabricated a type of intrinsic self-healing, highly conductive and stretchable hydrogels towards flexible strain sensors. Specifically, CNFs and GN were introduced to form GN-CNF nanocomplexes, which achieved the homogeneous distribution of GN in the PVA gel network. The incorporation of GN-CNF nanocomplexes considerably improved the electro-conductibility, elasticity and mechanical strength for the GN-CNF@PVA hydrogels. The hydrogen bonding system, entangling of PVA and GN-CNF, and dynamic and reversible multi-complexation that resulted from borax cross-linking contributed to the construction of the combined reinforcing and conductive network within the composite hydrogels. The strain sensors assembled by the GN-CNF@PVA hydrogel 
could sense and monitor the real-time body motion, demonstrating potential application in wearable sensing devices.

\section{Materials and Methods}

\subsection{Materials}

Sulfuric acid (98 wt\%, Nanjing Chemical Reagent) was adjusted to $48 \mathrm{wt} \%$ concentration and cellulose powder was dried for $12 \mathrm{~h}$ at $50{ }^{\circ} \mathrm{C}$ before use. Sodium tetraborate decahydrate (borax, $\geq 99.5 \%$ ) and polyvinyl alcohol (PVA, 99\% hydrolyzed, $M_{\mathrm{W}}=146,000 \sim 186,000 \mathrm{~g} \mathrm{~mol}^{-1}$ ) and were obtained from Sigma Chemical. Graphene (GN) was provided by Nanjing Kefu Nano-Tech Co. Ltd (Nanjing, China).

\subsection{Isolation of Cellulose Nanofibers (CNFs) and Synthesis of Graphene-CNF/Poly (Vinyl Alcohol) (GN-CNF@PVA) Hybrid Hydrogels}

In our previous work, cellulose nanofibers (CNFs) were isolated from cellulose powder by a combined acid hydrolysis and ultrasonification treatment [29,30]. The cellulose powder was hydrolyzed by using $48 \%$ by weight of sulfuric acid, wherein the ratio of acid to fibers was $20: 1$. The cellulose/acid solution was vigorously agitated at $45^{\circ} \mathrm{C}$ for $1 \mathrm{~h}$, and then the reaction was stopped by diluting 15 -fold with cold water. The mixture was filtered under vacuum several times and the slurry obtained was re-dispersed in water. The excess acid was removed by centrifugation at room temperature for 25 min at $12,000 \mathrm{rpm}$, then the water was dialyzed in a dialysis tube with a molecular weight of $12,000-14,000$ to $\mathrm{pH}=7$. To further improve the dispersibility, the CNFs aqueous suspension was sonicated in a 0.8 $\mathrm{kW}$ ultrasonic cell disrupter for $150 \mathrm{~min}$ to separate $\mathrm{CNF}$ at $0{ }^{\circ} \mathrm{C}$. Finally, the concentration of $\mathrm{CNF}$ suspension was controlled at $1.0 \mathrm{wt} \%$.

The GN-CNF@PVA hybrid hydrogels were prepared as follows. Firstly, the different amounts of graphene (GN) were slowly added into $200 \mathrm{~g}$ of CNF dispersion (1.0 wt \%, containing $2 \mathrm{~g}$ of CNF) to form suspension, which was then stirred and sonicated at $300 \mathrm{~W}$ for $15 \mathrm{~min}$ at room temperature. The aqueous suspension containing GN-CNF nanocomplexes was evaporated to a weight of $80 \mathrm{~g}$ in a rotary evaporator. Specifically, the weight proportion of GN to CNFs were fixed at 0.15:1, 0.25:1 and 0.35:1, and the GN-CNF counterparts were designated as GN-CNF-A, GN-CNF-B and GN-CNF-C nanocomplexes, respectively. Meanwhile, $2.0 \mathrm{~g}$ of polyvinyl alcohol (PVA) was dissolved and stirred in $20 \mathrm{~mL}$ of deionized water at $90^{\circ} \mathrm{C}$. After a complete dissolution of PVA, the dissolved PVA solution was slowly added to $80 \mathrm{~g}$ of GN-CNF aqueous suspension, and stirring was continued for $0.5 \mathrm{~h}$ at $90^{\circ} \mathrm{C}$. Finally, $0.4 \mathrm{~g}$ of sodium tetraborate decahydrate was dispersed into the mixture under agitation. After the borax was completely dissolved, the reaction system was cooled to room temperature to form GN-CNF@PVA hybrid hydrogels. The hydrogels with the weight proportion (GN to CNFs) of 0.15:1, 0.25:1 and 0.35:1 were designated as GN-CNF@PVA-A, GN-CNF@PVA-B and GN-CNF@PVA-C, respectively (Table 1). As a reference, pure PVA hydrogels and CNF-PVA hydrogels were synthesized via the same procedure in the absence of CNFs or GN.

Table 1. The ratio of substance in hydrogels.

\begin{tabular}{cccc}
\hline Sample & GN & CNF & PVA \\
\hline PVA & $0.0 \%$ & $0.0 \%$ & $2.0 \%$ \\
CNF/PVA & $0.0 \%$ & $2.0 \%$ & $2.0 \%$ \\
GN-CNF@PVA-A & $0.3 \%$ & $2.0 \%$ & $2.0 \%$ \\
GN-CNF@PVA-B & $0.5 \%$ & $2.0 \%$ & $2.0 \%$ \\
GN-CNF@PVA-C & $0.7 \%$ & $2.0 \%$ & $2.0 \%$ \\
\hline
\end{tabular}




\subsection{Characterizations}

Hydrogel densities $\left(\rho, \mathrm{g} \mathrm{cm}^{-3}\right)$ were calculated from the weight and size of the specimens. Hydrogel specimens (initial weight $\left.=W_{\mathrm{i}}\right)$ were dried at $45{ }^{\circ} \mathrm{C}$ to a steady weight $\left(W_{\mathrm{d}}\right)$. The moisture content $\left(W_{\mathrm{c}}\right)$ was obtained from Equation (1):

$$
W_{c}=\frac{W_{i}-W_{d}}{W_{i}} \times 100 \%
$$

The morphology of CNFs, GN and GN-CNF dispersions were analyzed on a transmission electron microscope (TEM, JEM-1400, JEOL, Tokyo, Japan) at $80 \mathrm{kV}$. The gel morphology was investigated by a scanning electron microscope (SEM, JSM-7600F, Tokyo, Japan) at $5.0 \mathrm{kV}$. Fourier transform infrared (FTIR) analysis was conducted using a spectrophotometer (Thermo Fisher, San Jose, CA, USA) at a $4 \mathrm{~cm}^{-1}$ resolution from 4000 to $600 \mathrm{~cm}^{-1}$. X-ray diffraction (XRD) analysis of GN, CNFs, PVA and GN-CNF@PVA was performed on an X-ray diffractometer (Ultima IV, Japan) at $40.0 \mathrm{kV}$ and $2 \theta=5 \sim 40^{\circ}$. The rheological viscoelastic parameters, such as loss modulus $\left(G^{\prime \prime}\right)$ and storage modulus $\left(G^{\prime}\right)$ versus $\omega$ were tested through the angular frequency $\left(\omega=0.1\right.$ to $\left.100 \mathrm{rad} \mathrm{s}^{-1}\right)$ at $\gamma=1 \%$ at room temperature. The complex modulus $\left(G^{*}\right)$ were approached by Equation (2).

$$
G^{*}=\sqrt{G^{\prime 2}+G^{\prime \prime 2}}
$$

Compression tests were carried out on the hydrogel cylinders (height $10.0 \mathrm{~mm}$ and diameter $35.0 \mathrm{~mm}$ ) by a test machine (SANS CMT4304, Sans Testing Machine, Shen Zhen, China) at $10.0 \mathrm{~mm} \mathrm{~min} \mathrm{~m}^{-1}$ compression speed. The uniaxial tensile measurements were conducted on the square specimens $\left(50 \times 5 \times 5 \mathrm{~mm}^{3}\right)$ with a stretching rate of $20.0 \mathrm{~mm} \mathrm{~min}^{-1}$, and the original distance between two clamps $\left(L_{0}\right)$ was fixed at $20.0 \mathrm{~mm}$. The tensile stress $\left(\sigma_{\mathrm{t}}\right)$ and compressive stress $\left(\sigma_{\mathrm{e}}\right)$ were approached by dividing the force $(F)$ by the original cross-sectional area $\left(A_{0}\right)$ of samples, while tensile strain $\left(\varepsilon_{t}\right)$ was obtained from the ratio of length $(L)$ to the original length $\left(L_{0}\right)$. The elastic modulus $(E)$ of the hydrogels was defined as the slope of the tensile stress-strain curve at $10-15 \%$ strain. The $\sigma$ and $\varepsilon$ values were calculated by Equations (3) and (4), respectively:

$$
\begin{gathered}
\sigma_{t, e}=F / A_{0} \\
\varepsilon_{t}=\left(L-L_{0}\right) / L_{0} \times 100 \%
\end{gathered}
$$

The conductivity measurements were performed on a CHI760E electrochemical workstation (Chenhua, Shanghai). Specifically, the gel specimens $\left(1.0 \times 0.5 \times 0.5 \mathrm{~cm}^{3}\right)$ were contained between two Pt electrodes. The hydrogel resistance was by Equation (5):

$$
R=U I
$$

where $R, U$ and $I$ were the hydrogel resistance $(\Omega)$, the potential $(V)$ and the current of open circuit (A), respectively.

The conductivities of the gel samples were approached by Equation (6):

$$
\sigma=\frac{1}{R} \frac{L}{S}
$$

where $L$ was the thickness of hydrogel samples $(\mathrm{cm}), S$ was the area of hydrogel samples $\left(\mathrm{cm}^{2}\right)$ and $R$ was the resistance of hydrogel samples $(\Omega)$.

To assess the self-healing property, square gel specimens were first cut into parts, which were immediately brought into contact in air without stimulation. After a certain time of the healing process, the tensile tests were performed to assess the self-healing performance. The healing efficiency of the elongation at break $(f)$ was calculated from the maximum elongation at break ratio of healed hydrogels 
$\left(\varepsilon^{\prime}\right)$ to the original ones $(\varepsilon)$ by Equation (7), where $\varepsilon^{\prime}$ and $\varepsilon$ were defined as dividing the original length by the elongation of the healed and original hydrogels during stretching, respectively.

$$
f=\varepsilon \prime / \varepsilon \times 100 \%
$$

The strain-sensing performance of hydrogel-based sensors were evaluated using an electrochemical workstation at $8.0 \mathrm{~V}$. A homemade stretching device was used to stretch the sensors ( $40 \mathrm{~mm}$ in length and $4 \mathrm{~mm}$ in diameter) to a certain strain.

\section{Results and Discussion}

\subsection{Fabrication Process of GN-CNF@PVA Composite Hydrogels}

The fabrication process of the GN-CNF@PVA hydrogel is presented in Figure 1a. The homogeneous $\mathrm{CNF}$ dispersion was extracted by a combined hydrolysis and sonication treatment. Because of their ideal dispersibility and small size, CNFs were used as an appropriate nanocarrier for promoting the dispersion of GN in aqueous medium, forming the interconnected conductive pathways. Subsequently, in the absence of any surfactant, the homogeneously dispersed GN-CNF nanocomplexes were distributed into the PVA polymer network to construct the GN-CNF@PVA hydrogel with 3D conducting pathways. During the gelation process, borax was quickly decomposed into $\mathrm{B}(\mathrm{OH})_{4}{ }^{-}$and $\mathrm{B}(\mathrm{OH})_{3}$ after being dissolved in water (Figure 1b). Among them, the multi-complexation occurred through the attachment of borax to neighboring $-\mathrm{OH}$ groups of the PVA and CNFs [24]. The tetrahedral $\mathrm{B}(\mathrm{OH})_{4}{ }^{-}$ion with four -OH groups at each corner could be reversibly complexed with cis-diol sites of GN-CNF nanocomplexes in water and PVA chains. Consequently, with the presence of borax cross-linker, GN-CNF nanocomplexes and PVA chains could be dynamically and reversibly complexed together. The multi-complexation, hydrogen bonding system and the entanglement of polymer chains successfully established a hierarchical 3D hydrogel network. As shown in Figure 1c, the GN-CNF@PVA gels obtained with a high water content $(\sim 95 \%)$ and a small density $\left(\sim 1.5 \mathrm{~g} \mathrm{~cm}^{-3}\right)$ exhibited a smooth surface. These free-standing hydrogels with an electro-conductivity $\left(\sim 3.55 \mathrm{~S} \mathrm{~m}^{-1}\right)$ could be readily molded into various shapes.

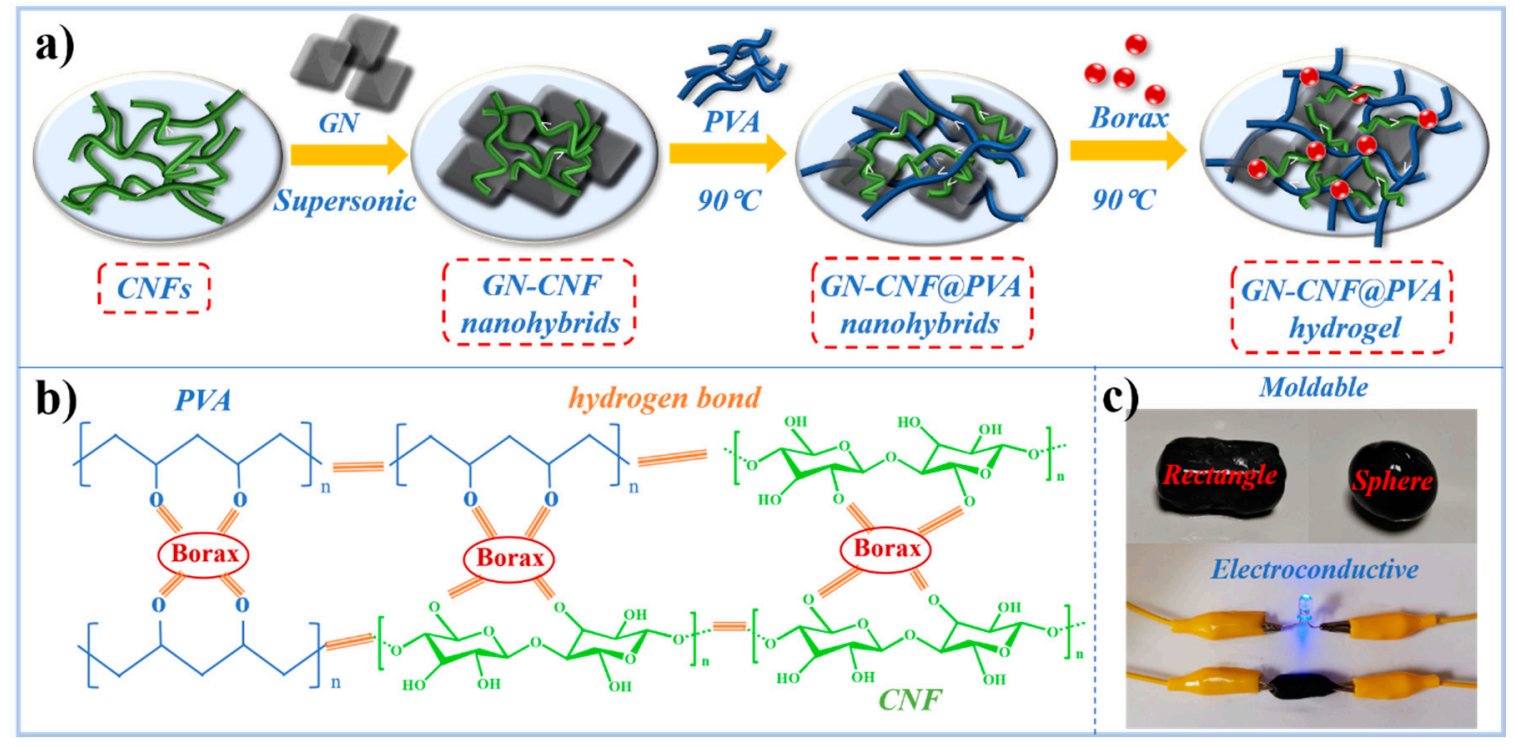

Figure 1. (a) Preparation process of graphene-cellulose nanofiber/poly (vinyl alcohol) (GN-CNF@PVA) hydrogels; (b) synthesis mechanism of the hydrogel network; (c) demonstration of the malleability and electro-conductivity of hydrogels. 


\subsection{Morphology of GN-CNF Nanocomplexes}

As shown in the TEM images of Figure 2a, CNFs with a high aspect ratio exhibited an average diameter and length of $28 \pm 8 \mathrm{~nm}$ and $653 \pm 73 \mathrm{~nm}$, respectively, forming a homogeneous colloid in water. GN exhibited a typical lamellar structure and agglomerated overlap state in water mainly because of the intermolecular stacking attraction and van der Waals interactions (Figure 2b), indicating the poor dispersity of pure GN in aqueous medium [31]. The morphology characteristics of GN-CNF nanocomplexes can be clearly observed in Figure 2c. Obviously, the GN aggregates were divided into thinner GN layers without significant overlap in the large area. Because of the electrostatic repulsion behavior of CNFs, the hydrophobic interactions between specific crystalline surfaces on the CNFs and GN, CNFs were capable of uniformly dispersing GN in water [32,33]. As shown in Figure 2d, the pure GN and GN-CNF nanocomplexes were dispersed well after $1 \mathrm{~h}$ of stirring. However, after $12 \mathrm{~h}$, due to the inherent intermolecular interactions, the pure GN formed a large-scale aggregation and precipitated in water, while the GN-CNF nanocomplexes remained homogeneously dispersed in water, further demonstrating the dispersing ability of CNFs for GN in water.
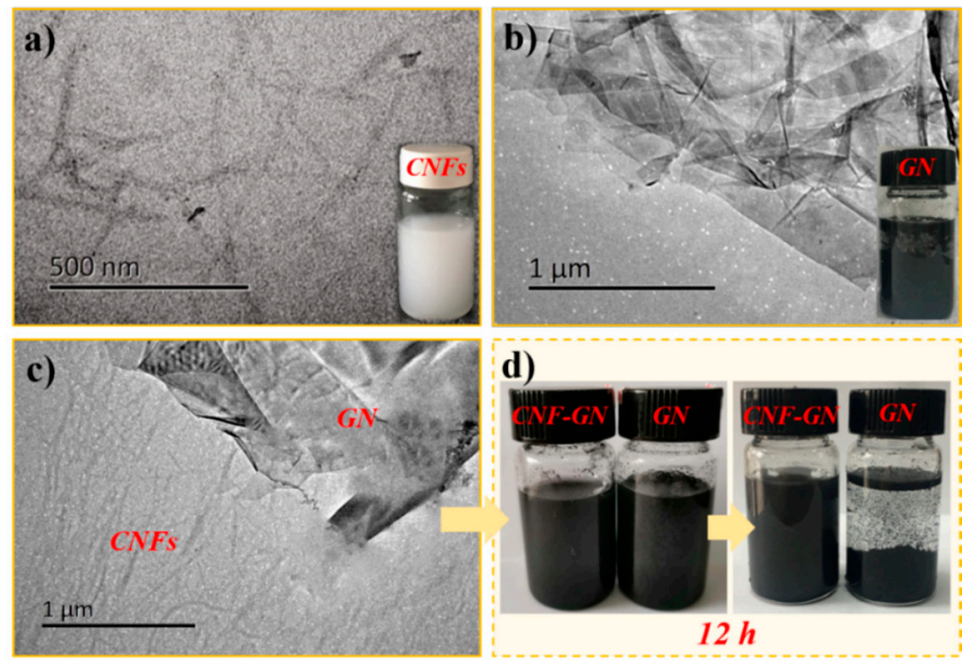

Figure 2. Transmission electron microscope (TEM) images of CNFs (a), GN (b), and GN-CNF nanocomplexes (c); (d) macroscopic pictures of pure GN and GN-CNF dispersions after a $12 \mathrm{~h}$ resting.

\subsection{Chemical Structure of GN-CNF@PVA Composite Hydrogels}

Figure 3a displays the FTIR curves of GN, CNFs, PVA hydrogel and GN-CNF@PVA hydrogel. Due to the defects of graphitic structure, the absorptions of $\mathrm{CH}_{2}$ and $\mathrm{CH}_{3}$ groups occurred at 2928 and $2969 \mathrm{~cm}^{-1}$, respectively [34]. CNFs showed representative peaks at 1079, 1720, 2977 and 3494 $\mathrm{cm}^{-1}$, due to $\mathrm{C}-\mathrm{H}$ bending, $-\mathrm{CH}_{2}$ in-plane bending, $\mathrm{C}-\mathrm{H}$ stretching and $\mathrm{O}-\mathrm{H}$ stretching vibration, respectively [35]. For the pure PVA hydrogel, the absorptions at 1350 and $1470 \mathrm{~cm}^{-1}$ corresponding to the $\mathrm{B}-\mathrm{O}-\mathrm{C}$ (stretching relaxation) verified the crosslinked network of borax and PVA [36]. When the GN-CNF nanocomplexes were distributed into PVA network, most featured peaks of cellulose overlapped with others. The absorption appeared at $2900 \mathrm{~cm}^{-1}$ due to the $\mathrm{C}-\mathrm{H}$ symmetric stretching of methylene group [37]. Compared to the pure PVA gel, the featured absorptions of the hydrogen bonds at $3494 \mathrm{~cm}^{-1}$ and $3486 \mathrm{~cm}^{-1}$ moved from the higher wave zone to the lower wave zone, revealing the strong linkage between the PVA and the GN-CNF nanocomplexes [38]. As shown in the XRD patterns of Figure $3 b$, the pure GN had a sharp diffraction peak at $2 \theta=26.5^{\circ}$ [39]. CNFs presented a prominent peak at $2 \theta=22.0^{\circ}$ and a lower overlapped peak at $2 \theta=15.1^{\circ}$ and $16.2^{\circ}$, corresponding to (101) and (002) planes [29]. PVA hydrogel exhibited a diffraction peak at $2 \theta=19.4^{\circ}$ [40]. For the GN-CNF@PVA hydrogel, the characteristic peak of PVA vanished, and the featured peaks of GN and CNFs were slightly shifted to $2 \theta=25.6^{\circ}$ and $22.1^{\circ}$. These results illustrated the dynamic interaction between borate ions, PVA and GN-CNF nanocomplexes [41]. 

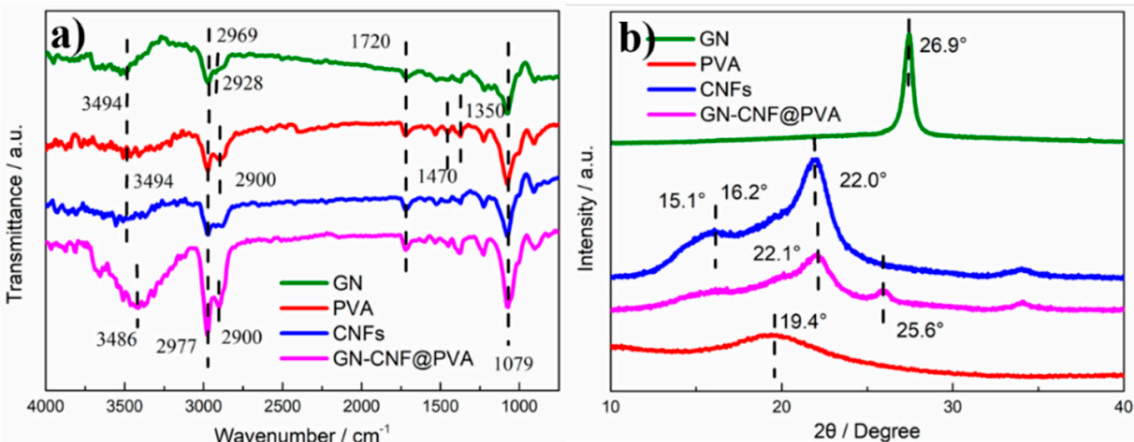

Figure 3. (a) Fourier transform infrared (FTIR) analysis and (b) X-ray diffraction (XRD) results of GN, CNFs, PVA hydrogel and GN-CNF@PVA hydrogel.

\subsection{Rheological Behavior of Composite Hydrogels}

The dynamic oscillatory frequency sweep measurements of all hydrogels were tested at $\omega=0.1 \sim 100 \mathrm{rad} \mathrm{s}^{-1}$ (Figure 4a). Within the entire frequency span, G" were generally lower than $\mathrm{G}^{\prime}$, revealing the hydrogels had a fairly stable and strong permanent network [42]. The hydrogel viscoelasticity was improved with the integration of GN-CNF nanocomplexes. Compared with pure PVA and CNF/PVA gels, the $G_{\infty}^{\prime}$ (high-frequency plateau of $G^{\prime}$ ) value of GN-CNF@PVA-B hydrogel was increased by almost 5-fold and 9-fold, respectively. Among all hydrogels, the GN-CNF@PVA-B had the highest $\mathrm{G}^{\prime}(\sim 3700.5 \mathrm{~Pa})$ and $\mathrm{G}^{\prime \prime}(\sim 770.4 \mathrm{~Pa})$ values (Table 2$)$. This indicated strong interaction and entanglement between the PVA chain and the GN-CNF nanocomposite, as well as a more stable and higher crosslink [38]. When the GN content reached $0.7 \mathrm{wt} \%$, the excessive GN affected the cross-linking degree at polymer intersections and hindered the creation of the hydrogel network, leading to the decrease of viscoelasticity. Figure $4 \mathrm{~b}$ provided a more significant contrast of all hydrogels, where GN-CNF@PVA-B hydrogel possess the highest $\mathrm{G}^{*}(\sim 4227.8 \mathrm{~Pa})$ within the whole $\omega$ range.
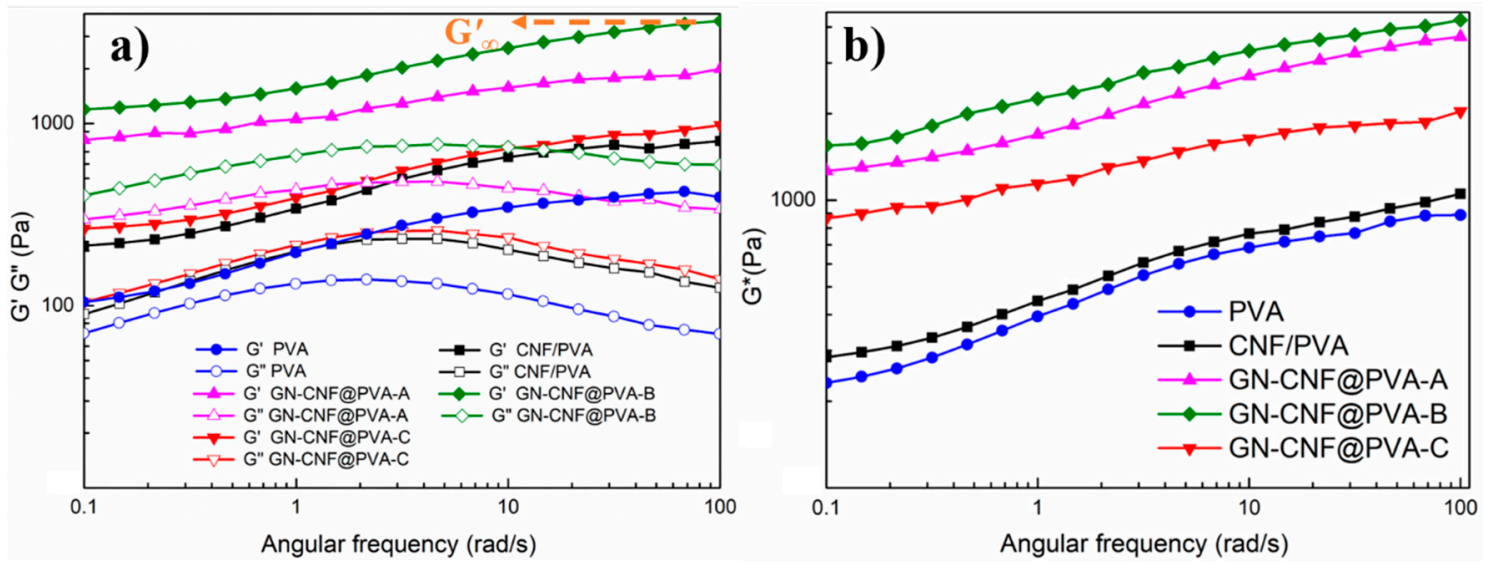

Figure 4. Rheological behavior of different gels under room temperature. (a) $G^{\prime}$ and $G^{\prime \prime}$ versus $\omega$; (b) frequency dependence of $G^{*}$ versus $\omega$.

Table 2. Viscoelastic properties of hydrogels obtained from moduli plots.

\begin{tabular}{cccccc}
\hline Parameter & PVA & CNF/PVA & GN-CNF@PVA-A & GN-CNF@PVA-B & GN-CNF@PVA-C \\
\hline High-frequency & 421.1 & 790.1 & 1989.8 & 3700.5 & 973.3 \\
plateau of $G^{\prime}, G_{\infty}^{\prime}(\mathrm{Pa})$ & 141.3 & 237.6 & 471.3 & 770.4 & 252.9 \\
Maximum $G^{\prime \prime}, G^{\prime \prime}(\mathrm{Pa})$ & 1051.0 & 3698.0 & 4227.8 & 2032.4 \\
Maximum $G^{*}, G^{*}(\mathrm{~Pa})$ & 887.7 & 10.0 & \\
\hline
\end{tabular}




\subsection{Mechanical Properties of GN-CNF@PVA Hybrid Hydrogels}

The mechanical strength of hydrogels were comparatively evaluated by the energy absorption $\left(\mathrm{E}_{\mathrm{a}}\right)$ and the compressive stress $\left(\sigma_{\mathrm{e}}\right)$ at $\varepsilon=75 \%$ (Figure 5a,b). Overall, GN-CNF@PVA-B hydrogels sustained higher stress than the other hydrogels. Among them, the $\sigma_{\mathrm{e}}$ values of CNF/PVA hydrogel $(\sim 15.3 \pm 1.2 \mathrm{KPa})$ was about 2-fold higher than pure PVA gel $(\sim 7.4 \pm 0.7 \mathrm{KPa})$, indicating that CNFs considerably improved the mechanical toughness of hydrogels. The homogeneously distributed GN-CNF nanocomplexes in a borax-crosslinked PVA gel network contributed to transferring the stress from polymer chains to GN-CNF nanocomplexes and halting the development of microcracks, thus resulting in the promotion in the hydrogel strength [43]. The $\sigma_{e}$ values of GN-CNF@PVA-B was about 10 times greater than CNF/PVA hydrogels. One possible reason was that the $\mathrm{B}(\mathrm{OH})^{4-}$ ions were capable of reversibly complexing with the cis-diol sites of the GN-CNF nanocomplexes and PVA, and the entangling of the CNFs and PVA chains could also promote the establishment of the 3D gel network with a stronger structure [36]. In addition, when the content of GN was $0.7 \mathrm{wt} \%$, the $\sigma_{\mathrm{e}}$ values of GN-CNF@PVA-C hydrogel was lower than the other hydrogels, indicating that the excess GN could reduce the effective crosslinking of GN-CNF nanocomplexes and PVA matrix. The energy absorption-strain curves displayed a more obvious contrast of all the gels in Figure $5 \mathrm{~b}$. The GN-CNF@PVA-B hydrogel reached an $E_{a}$ value of $1.8 \mathrm{~kJ} \mathrm{~m}^{-3}$ at the $75 \%$ strain level (Table 3), which further demonstrated that GN-CNF nanocomplexes could reinforce compressive performance of hydrogels. As displayed in the SEM of Figure 5c, the porous structure of GN-CNF@PVA hydrogel confirmed the 3D interconnected network, and the GN-CNF nanocomplexes was uniformly dispersed in the hydrogel, which contributed to the enhancement of mechanical strength.
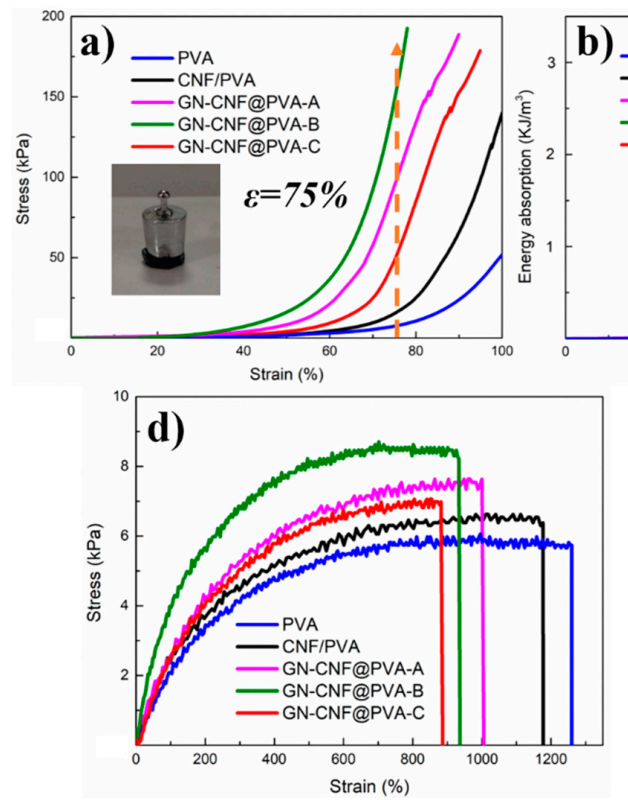

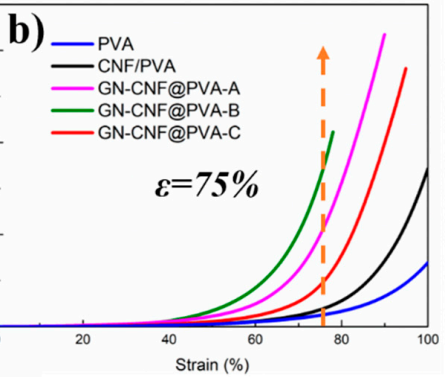

Strain (\%)

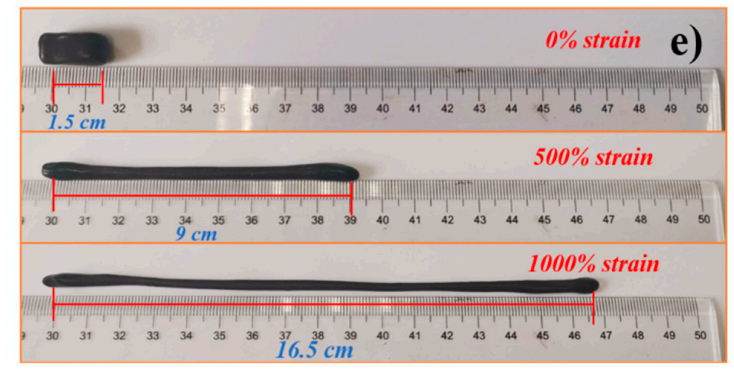

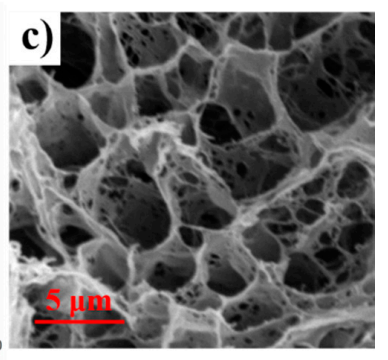

))

Figure 5. (a) Compression and (b) energy absorption graphs of various gels; (c) scanning electron microscope (SEM) image of GN-CNF@PVA-B hydrogel; (d) tensile graphs of PVA, CNF/PVA and GN-CNF@PVA gels; (e) demonstration of the stretchability of the GN-CNF@PVA-A gel.

Table 3. Physical-mechanical performances of original gels.

\begin{tabular}{|c|c|c|c|c|c|c|c|}
\hline Hydrogels & $\begin{array}{c}\sigma_{e} \text { at } \varepsilon=75 \% \\
(\mathrm{KPa})\end{array}$ & $\begin{array}{c}E_{\mathrm{a}} \text { at } \varepsilon=75 \% \\
\left(\mathrm{~kJ} \mathrm{~m}^{-3}\right)\end{array}$ & $\sigma_{t}(\mathrm{KPa})$ & $\varepsilon_{t}(\%)$ & $E(\mathrm{KPa})$ & $W_{\mathrm{c}}(\%)$ & $P\left(\mathrm{~g} \mathrm{~cm}^{-3}\right)$ \\
\hline PVA & $7.4 \pm 0.7$ & $0.2 \pm 0.1$ & $5.9 \pm 0.3$ & $1264.3 \pm 50.2$ & $4.1 \pm 0.5$ & $96.3 \pm 0.2$ & $1.0 \pm 0.1$ \\
\hline CNF/PVA & $15.3 \pm 1.2$ & $0.3 \pm 0.1$ & $6.6 \pm 0.6$ & $1177.7 \pm 35.7$ & $4.9 \pm 0.8$ & $95.5 \pm 0.4$ & $1.2 \pm 0.3$ \\
\hline GN-CNF@PVA-A & $95.0 \pm 8.3$ & $1.0 \pm 0.3$ & $7.6 \pm 0.5$ & $1000.2 \pm 36.8$ & $6.3 \pm 1.0$ & $95.2 \pm 0.1$ & $1.3 \pm 0.1$ \\
\hline GN-CNF@PVA-B & $148.1 \pm 10.4$ & $1.8 \pm 0.4$ & $8.5 \pm 0.4$ & $936.7 \pm 23.5$ & $9.4 \pm 1.2$ & $95.0 \pm 0.2$ & $1.5 \pm 0.2$ \\
\hline GN-CNF@PVA-C & $48.0 \pm 3.5$ & $0.5 \pm 0.2$ & $7.1 \pm 0.3$ & $866.6 \pm 28.4$ & $5.2 \pm 0.4$ & $94.7 \pm 0.3$ & $1.6 \pm 0.2$ \\
\hline
\end{tabular}


The tensile stress-strain graphs for different hydrogel samples are presented in Figure $5 \mathrm{~d}$. The incorporation of GN-CNF nanocomplexes considerably enhanced the tensile stress $\left(\sigma_{t}\right)$ of the gels. Pure PVA hydrogel showed the lowest $\sigma_{t}$ value $(\sim 5.9 \pm 0.3 \mathrm{KPa})$, while the GN-CNF@PVA-B hydrogel $(\sim 8.5 \pm 0.4 \mathrm{KPa})$ had the highest $\sigma_{\mathrm{t}}$ value. As the content of GN-CNF nanocomplexes rose, the $\sigma_{\mathrm{t}}$ were gradually enlarged, while the elongation at break $\left(\varepsilon_{\mathrm{t}}\right)$ were gradually declined. The $\varepsilon_{\mathrm{t}}$ of the original PVA hydrogel reached $1264.3 \pm 50.2 \%$, but the GN-CNF@PVA-B hydrogel decreased to 936.7 $\pm 23.5 \%$. The well-dispersed GN-CNF nanocomplexes could interact with the PVA matrix, forming plenty of cross-linking points with PVA. Furthermore, the hydrogel network could dynamically break and recombine owing to borax, and thus GN-CNF nanocomplexes could act as effective reinforcement to improve the hydrogel strength [44]. The decrease of elongation at break resulted from the increased interaction between PVA chains and GN-CNF nanocomplexes, which hindered the migration of the PVA chains [45]. When the GN content was above $0.5 \mathrm{wt} \%$, both the $\sigma_{\mathrm{t}}$ and the $\varepsilon_{\mathrm{t}}$ values were decreased. This result indicated that the addition of excess GN caused the formation of aggregates and denser polymer network, which had a severe adverse effect on the toughness and stretchability of the hydrogel [39]. In addition, the elongation at yield strength and yield strength were also two important indexes for hydrogels in tensile properties. As shown in Table S1, GN-CNF@PVA-B hydrogel demonstrated the highest yield strength $(\sim 8.5 \mathrm{kPa})$, which was consistent with the above results. Overall, GN-CNF@PVA-B hydrogel had the largest tensile properties. The high tensile properties of the hydrogel are more intuitively demonstrated with a tensile deformation of about $1000 \%$, as shown in Figure 5e. Although these hydrogels exhibited good tensile properties, there were still stretchability limitations for their application in wearable e-skin devices due to the plastic deforming at low strains.

\subsection{Self-Healing Properties of GN-CNF@PVA Hybrid Hydrogels}

Figure 6 demonstrates the self-healing performance of these gels. Two freshly prepared GN-CNF@PVA and CNF/PVA hydrogels were put together (Figure 6a). After contact for $20 \mathrm{~s}$, their surfaces were merged together and autonomously self-healed to a monolithic hydrogel without stimulation, revealing a rapid and intrinsic self-restore capability. The self-healed hydrogels could even be stretched without damage at the contacting interface. The moldable hydrogels with "NFU" shape still exhibited excellent self-healing ability when being connected and even lifted (Figure $6 \mathrm{~b}$ ). The ideal self-healing behavior with GN-CNF@PVA hydrogels resulted from the hydrogen bonding system, the dynamic and reversible multi-complexation between -OH groups of GN-CNF, PVA and borax [38]. The self-healing mechanism of hydrogels is shown in Figure 6c. These hydrogen bonds could be readily damaged and re-established, and thus the hydrogel could be remoulded and reformed repeatedly, endowing the hydrogels with intrinsic self-repairing ability and malleability at room temperature [24]. To explore the repeatability of in situ self-healing behavior, the GN-CNF@PVA-B hydrogels were circularly split at identical locations and repeatedly self-healed under constant conditions three times. Figure $6 \mathrm{~d}$ presents the stress-strain plots of the original and restored hydrogels after various cutting and healing loops, and the corresponding healing efficiencies are presented in Figure 6e. The 1st, 2nd and 3rd healing efficiency reached up to $97.7 \pm 1.2 \%, 96.1 \pm 1.4 \%$, and $94.8 \pm 1.0 \%$, respectively. The high healing efficiency was ascribed to the excellent healing repeatability of GN-CNF@PVA-B hydrogels. Nevertheless, a slight decline in self-healing efficiency value could not be avoided, because a small number of covalent bonds in neighboring polymer chains were permanently destroyed during the damage [46].

\subsection{Electrochemical Performance of Hydrogels}

Figure 7a shows the electro-conductivity change of GN-CNF@PVA with various content of GN. The electrical conductivities for all hydrogels presented a non-linear rise as the GN amount increased. At $\mathrm{GN}=0.7 \mathrm{wt} \%$, an electro-conductibility of $3.55 \mathrm{~S} \cdot \mathrm{m}^{-1}$ was generated for GN-CNF@PVA. As reported previously, CNFs could endow pathways for ion and electron transporting with their electrochemical hybrids with other materials [47]. Consequently, the $1 \mathrm{~d}$ configuration of CNFs allowed less percolation 
concentration of GN-CNF nanocomplexes to accomplish a continued conducting network, and the homogeneously dispersed GN-CNF nanocomplexes additionally created efficient conducting paths inside GN-CNF@PVA hydrogels [35]. Besides, the multi-complexation among borate ions, PVA and GN-CNF nanocomplexes were capable of providing the hydrogels with conducting paths, thus facilitating the electronic transmission [48]. To clearly demonstrate the self-restoring capability of the conducting network inside hydrogels, GN-CNF@PVA hydrogel was connected to a closed circuit (Figure $7 \mathrm{~b}$ ). After the circuit was connected, the light-emitting diode (LED) bulb was immediately lit, suggesting the ideal electro-conductibility of the GN-CNF@PVA hydrogel with a hierarchical conducting network [46]. When the GN-CNF@PVA hydrogel was completely cut into two pieces, the blue bulb was extinguished under an open circuit. Once the two pieces self-healed, the LED indicator could be turned on again. Figure 7c displays the current variations over time for GN-CNF@PVA-B hydrogel during damaging and self-healing cycles. The electrical current of the self-healed hydrogel remained relatively stable under multiple cutting/healing cycles. The demonstration illustrated that the GN-CNF@PVA hydrogel had an ideal electrical self-healing capacity, suggesting many promising applications in the field of self-repairing soft electronics [24].
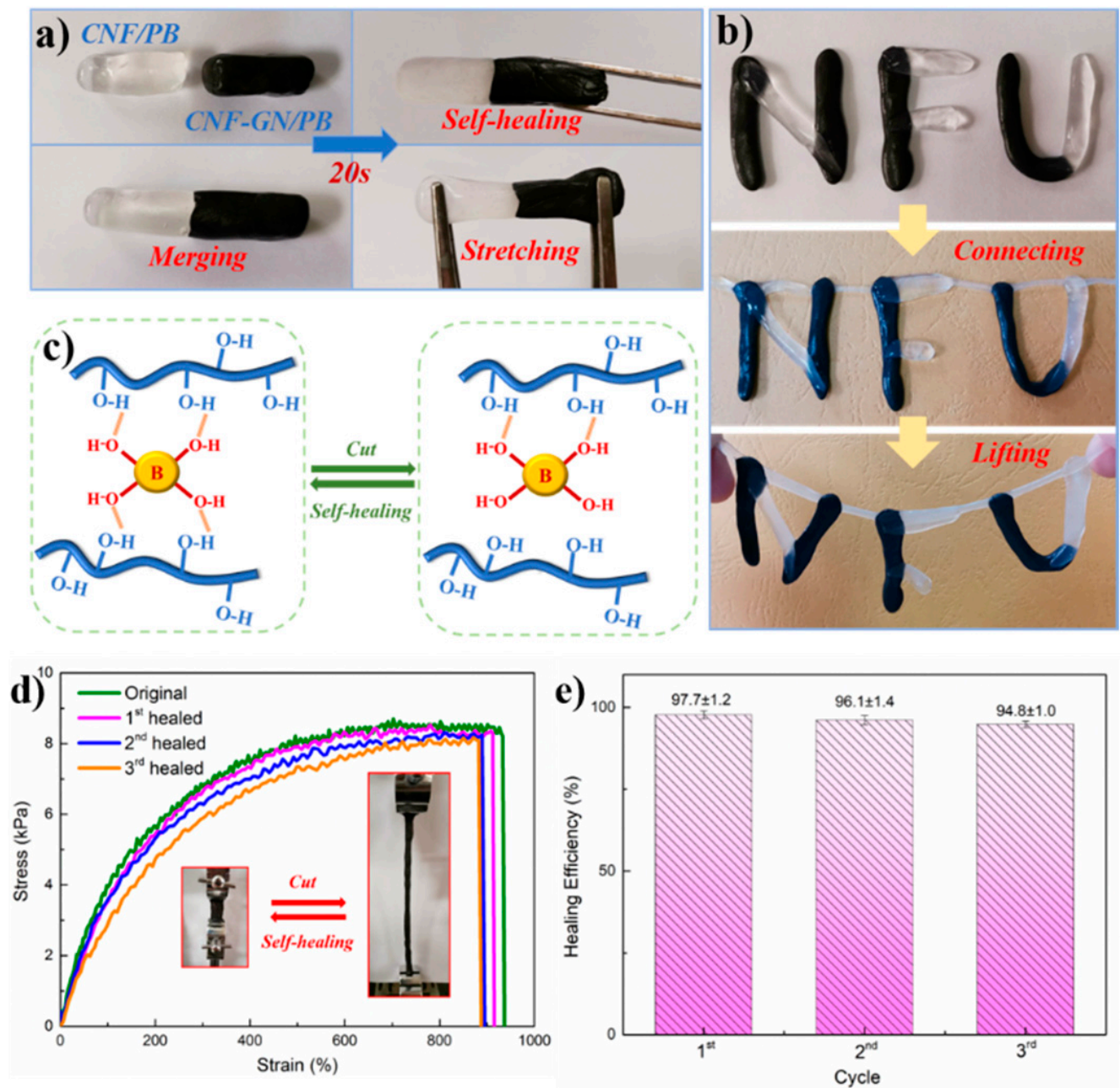

Figure 6. (a) The self-healing behavior of merging GN-CNF@PVA and CNF/PVA gels together; (b) photographs of the excellent healing ability in the different words for GN-CNF@PVA and CNF/PVA hydrogels; (c) self-healing mechanism of GN-CNF@PVA hydrogel; (d) stress-strain plots of original and self-healed GN-CNF@PVA-B hydrogels; (e) the values of healing efficiency in elongation at break after multiple breaking/healing cycles. 

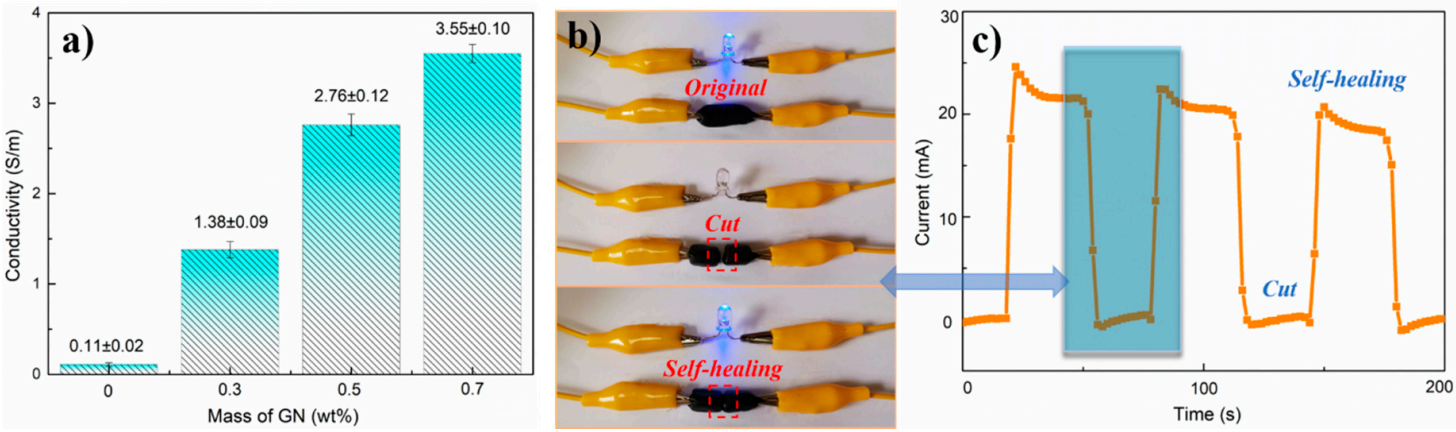

Figure 7. (a) Conductivity variation of GN-CNF@PVA gels with various amount of GN; (b) self-healing ability of conducting network in GN-CNF@PVA hydrogel when connected into a circuit; (c) current changes of the GN-CNF@PVA-B hydrogel during multiple cutting/healing cycles.

\subsection{Strain-Sensitive Performance of GN-CNF@PVA Hydrogel-Based Sensor}

To assess the practicability of GN-CNF@PVA gel for use in wearable sensing applications, the GN-CNF@PVA hydrogel embedded in a closed circuit equipped with a green bulb (Figure 8a). The illumination of the green bulb gradually deceased with the increasing applied strain $(0 \% \sim 500 \%)$, suggesting that the hydrogel electro-conductivity was highly sensitive to the applied strain [2]. As the applied tensile stress enhanced, the spaces between the conducting particles inside the hydrogel were enlarged, resulting in the increased resistance of hydrogels. This feature allowed these hydrogels to be used in the field of motion sensing. The strain sensitivity of the hydrogel-based sensors could be assessed quantitatively by the gauge factor (GF), calculated from the equation, $G F=\left(\left(R-R_{0}\right) / R_{0}\right) / \varepsilon$, where $\varepsilon$ was the strain, $R$ and $R_{0}$ were the resistance when stretching and the original resistance, respectively. From Figure $8 \mathrm{~b}$, it was found that GF values increased as the applied strain increased. When the strain increased to $500 \%$, the value of GF reached up to 3.8 , which was superior to various hydrogel-based strain sensors previously reported, such as the k-carrageenan/Polyacrylamide double-network hydrogels (GF 0.3 at 500\% strain) [49] and PVA-PVP hybrid hydrogels (GF 0.48 at $200 \%$ strain) [2]. In general, the GF values of hydrogel-based strain sensors increased with the increasing strain. Under a high strain level, the GF values of CNTs/PVA hydrogels [26] and Polyacrylamide-Polydopamine hydrogels [50] were 0.24 and 0.70 at $1000 \%$ strain, respectively, which were even lower than the GF value of GN-CNF@PVA hydrogels at 500\% strain. Such an ideal sensitivity endowed GN-CNF@PVA hydrogels with great potential in strain sensors. To demonstrate a real application of the GN-CNF@PVA hydrogel-based strain sensor, the sensor assembled by hydrogels was installed on a human index finger using conducting tapes, and then two cables were connected to an electrochemical workstation to monitor the variation of current (Figure 8c). When the finger was rapidly bent from $0^{\circ}$ to $90^{\circ}$, the current reduced gradually in response to the movements of the finger (Figure $8 \mathrm{~d}$ ), and the intensity variation of the current peak could accurately reflect the bending angle in real time [25]. Simultaneously, the displayed current variations exhibited practically constant peaks and shapes, indicating the repeatability, stability and reliability of the GN-CNF@PVA hydrogel-based strain sensors [51]. As shown in Figure 8e, more complicated hand motions could also be sensed easily by the assembled sensors. Each gesture for "one", "two", "three", "four" and "five" generated a different current response, and the current response was completely restorable. Interestingly, the current response remained steady under a certain gesture. Therefore, the current variation was capable of monitoring various hand gestures precisely in real time [14]. Once again, the GN-CNF@PVA hydrogel-based strain sensor had a remarkable electrical stability and repeatably in human-motion detection. 

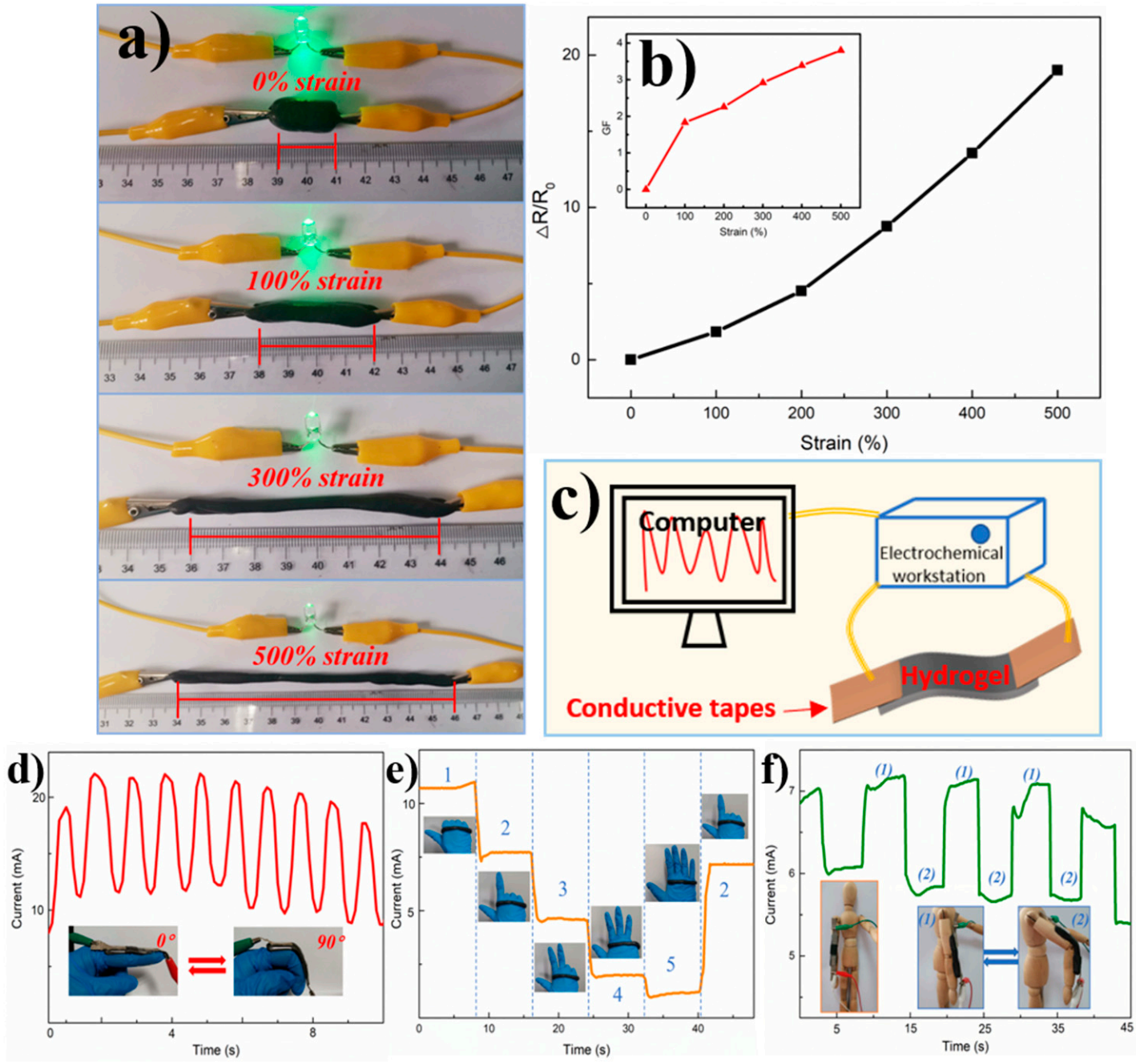

Figure 8. (a) Demonstration of the variations for light-emitting diode (LED) illumination vs different strain of the GN-CNF@PVA gels in a closed circuit; (b) the corresponding resistance variations due to the strain; (c) schematic illustration of strain sensor: current change of the GN-CNF@PVA hydrogels responding to rapidly finger bending (d) and various hand gestures (e); (f) the recorded current variations of the GN-CNF@PVA hydrogel-based strain sensor in arm bending.

The hydrogel-based strain sensor could further be attached onto a puppet to monitor the arm and leg movements (Figure $8 \mathrm{f}$ and Figure S1). During the arm and leg motions, the strain sensors were stretched to generate a tensile stress, which could destroy the conducting network and thus decrease the current values. More importantly, the bending and relaxation of the arms and legs could be easily distinguished based on the output current. The response behavior was repeatable, producing a similar current response by repeating the same motions [51,52]. Besides large-scale action, the hydrogel-based sensors could even detect some complex and unique waveforms as illustrated in Figure S1. For example, when handwriting the simple word "OK" or the shape of love on paper with the strain sensor, it was completely different from the response of current in the previously simple bending motion. It could be observed that the different motions exhibited different responses of current, resulting in the different waveforms. More importantly, when repetitive motion was applied, approximately constant response curves were produced, revealing an ideal reproducibility of these hydrogel-based strain sensors [53]. Therefore, the hydrogel-based strain sensor could not only distinguish simple human motions, but also identify complex spatial movements, which is beneficial to the further development of sign language recognition and communication of deaf-mute people [51]. 
GF and sensitivity were two important factors for hydrogel-based strain sensor. Many methods had been proposed to improve the GF values of strain sensors. For example, microcracks were introduced into the strain sensor at low strain, and the GF value could be increased quickly [54]. However, the low detection range of these strain sensors limited their application greatly. Then it was proposed to build a 3D conductive network and prepare a strain sensor with rough microstructure $[55,56]$. Even though the GF value was improved, the application of sensors was limited because it was difficult to completely contact with the skin. Therefore, fabrication of an ultra-thin sensor might be a promising way to increase GF values and sensitivity [57].

\section{Conclusions}

In conclusion, the current study prepared a novel type of highly stretchable, conductive and self-restorable hydrogel-based strain sensor, which was formed via dynamic and reversible multi-complexation between borax cross-linker, PVA matrix and GN-CNF nanocomplexes. Biomass-derived CNFs acts as templates and nanocarriers to support GN to create uniformly dispersed GN-CNF nanocomplexes in PVA gel matrix, enhancing the viscoelasticity, mechanical strength and electro-conductivity of the GN-CNF@PVA hydrogel. At an optimal content of $0.5 \mathrm{wt} \% \mathrm{GN}$, the GN-CNF@PVA-B hydrogel presented high stretchability (the elongation at break up to $1000 \%$ ), high compressive strength $(148.1 \pm 10.4 \mathrm{kPa})$, excellent viscoelasticity $\left(\mathrm{G}_{\max }^{\prime} \approx 3.7 \mathrm{kPa}\right)$ and ideal electro-conductivity (up to $3.55 \pm 0.1 \mathrm{~S} \mathrm{~m}^{-1}$ ). Due to the dynamic reversible bonds formed by borate ions with GN-CNF nanocomplexes and PVA chains, the hydrogel exhibited rapid a self-healing behavior (within $20 \mathrm{~s}$ ) with a high healing efficiency $(97.7 \pm 1.2 \%)$. Towards the practical wearable application, the strain sensor assembled by GN-CNF@PVA hydrogel was capable of effectively monitoring and distinguishing diverse human movements with a GF of about 3.8, demonstrating excellent sensitivity, repeatability and stability to the signals. Therefore, we expect the GN-CNF@PVA hydrogel-based strain sensor can attain promising applications in intelligent wearable electronics.

Supplementary Materials: The following are available online at http:/www.mdpi.com/2079-4991/9/7/937/s1. Figure S1: The recorded current variations of the GN-CNF@PVA hydrogel-based strain sensor in different motions: walking (a), handwriting the word "OK" (b), and handwriting the shape of love (c); Table S1: The yield index of original gels.

Author Contributions: C.Z. and Y.Y. contributed equally to this work. conceptualization, J.H.; methodology, L.G.; validation, Y.Y., X.X. and J.H.; formal analysis, C.M.; investigation, X.X.; resources, J.H.; writing-original draft preparation, C.Z.; writing-review and editing, J.H., L.G., X.X., C.M. and J.H.; supervision, J.H.

Funding: This research was funded by National Natural Science Funding of China (31770609), Natural Science Foundation of Jiangsu Province for Outstanding Young Scholars (BK20180090), 333 Project Foundation of Jiangsu Province (BRA2018337), Natural Science Research Project of Jiangsu Province (17KJB220007), Natural Science Foundation of Jiangsu Province (BK20160938), Qing Lan Project of Jiangsu Province (2019), Analysis and Test Center of Nanjing Forestry University, and Priority Academic Program Development of Jiangsu (PAPD).

Conflicts of Interest: The authors declare no conflict of interest.

\section{References}

1. Park, H.; Jeong, Y.R.; Yun, J.; Hong, S.Y.; Jin, S.; Lee, S.J.; Ha, J.S. Stretchable array of highly sensitive pressure sensors consisting of polyaniline nanofibers and Au-coated polydimethylsiloxane micropillars. ACS Nano 2015, 9, 9974-9985. [CrossRef] [PubMed]

2. Liu, Y.J.; Cao, W.T.; Ma, M.G.; Wan, P. Ultrasensitive Wearable Soft Strain Sensors of Conductive, Self-healing, and Elastic Hydrogels with Synergistic "Soft and Hard" Hybrid Networks. ACS Appl. Mater. Interfaces 2017, 9, 25559-25570. [CrossRef] [PubMed]

3. Kim, Y.; Zhu, J.; Yeom, B. Stretchable nanoparticle conductors with self-organized conductive pathways. Nature 2013, 500, 59-63. [CrossRef] [PubMed]

4. Lee, J.; Kim, S.; Lee, J. A stretchable strain sensor based on a metal nanoparticle thin film for human motion detection. Nanoscale 2014, 6, 11932-11939. [CrossRef] [PubMed] 
5. Zhang, M.; Wang, C.; Wang, H. Carbonized Cotton Fabric for High-Performance Wearable Strain Sensor. Adv. Funct. Mater. 2017, 27, 1604795. [CrossRef]

6. Wang, C.; Li, X.; Gao, E. Carbonized Silk Fabric for Ultrastretchable, Highly Sensitive, and Wearable Strain Sensors. Adv. Mater. 2016, 28, 6640-66408. [CrossRef] [PubMed]

7. Khan, H.; Razmjou, A.; Ebrahimi Warkiani, M. Sensitive and Flexible Polymeric Strain Sensor for Accurate Human Motion Monitoring. Sensors 2018, 18, 418. [CrossRef]

8. Yuan, C.; Lou, Z.; Wang, W. Synthesis of $\mathrm{Fe}_{3} \mathrm{C} @ \mathrm{C}$ from Pyrolysis of $\mathrm{Fe}_{3} \mathrm{O}_{4}$-Lignin Clusters and Its Application for Quick and Sensitive Detection of PrPSc through a Sandwich SPR Detection Assay. Int. J. Mol. Sci. 2019, 20, 741. [CrossRef]

9. Tang, G.; Xiong, R.; Lv, D. Gas-Shearing Fabrication of Multicompartmental Microspheres: A One-Step and Oil-Free Approach. Adv. Sci. 2019, 6, 1802342. [CrossRef]

10. Zhao, F.; Shi, Y.; Pan, L. Multifunctional Nanostructured Conductive Polymer Gels: Synthesis, Properties, and Applications. Acc. Chem. Res. 2017, 50, 1734-1743. [CrossRef]

11. Shao, C.; Wang, M.; Meng, L. Mussel-Inspired Cellulose Nanocomposite Tough Hydrogels with Synergistic Self-Healing, Adhesive, and Strain-Sensitive Properties. Chem. Mater. 2018, 30, 3110-3121. [CrossRef]

12. Chen, J.; Yu, Y.; Han, J. Mechanochemical esterification of waste mulberry wood by wet Ball-milling with tetrabutylammonium fluoride. Bioresour. Technol. 2019, 285, 121354. [CrossRef] [PubMed]

13. Zhang, L.L.; Lu, H.L.; Yu, J. Preparation of high-strength sustainable lignocellulose gels and their applications for anti-ultraviolet weathering and dye removal. ACS Sustain. Chem. Eng. 2019, 7, 2998-3009. [CrossRef]

14. Spoljaric, S.; Salminen, A.; Luong, N.D. Stable, self-healing hydrogels from nanofibrillated cellulose, poly(vinyl alcohol) and borax via reversible crosslinking. Eur. Polym. J. 2014, 56, 105-117. [CrossRef]

15. Wang, Z.; Kang, H.; Zhao, S.; Zhang, W.; Zhang, S. Polyphenol-induced cellulose nanofibrils anchored graphene oxide as nanohybrids for strong yet tough soy protein nanocomposites. Carbohydr. Polym. 2018, 180, 354-364. [CrossRef] [PubMed]

16. Zhang, X.; Pint, C.L.; Lee, M.H. Optically-and thermally-responsive programmable materials based on carbon nanotube-hydrogel polymer composites. Nano Lett. 2011, 11, 3239-3244. [CrossRef] [PubMed]

17. Yan, Q.; Li, J.; Zhang, X.; Zhang, J.; Cai, Z. Synthetic Bio-Graphene Based Nanomaterials through Different Iron Catalysts. Nanomaterials 2018, 8, 840. [CrossRef] [PubMed]

18. Chuang, W.; Chiu, W.; Tai, H. Temperature-dependent conductive composites: Poly (N-isopropylacrylamide-co-N-methylol acrylamide) and carbon black composite films. J. Mater. Chem. 2012, 22, 20311-20318. [CrossRef]

19. Tee, B.C.; Wang, C.; Allen, R. An electrically and mechanically self-healing composite with pressure- and flexion-sensitive properties for electronic skin applications. Nat. Nanotechnol. 2012, 7, 825-832. [CrossRef]

20. Choi, S.; Lee, H.; Ghaffari, R. Recent advances in flexible and stretchable bio-electronic devices integrated with nanomaterials. Adv. Mater. 2016, 28, 4203-4218. [CrossRef]

21. Naficy, S.; Razal, J.M.; Spinks, G.M. Electrically Conductive, Tough Hydrogels with pH Sensitivity. Chem. Mater. 2012, 24, 3425-3433. [CrossRef]

22. Bodenmann, A.K.; Macdonald, A.H. Graphene: Exploring carbon flatland. Phys. Today 2007, 60, 35-41. [CrossRef]

23. Cai, G.; Wang, J.; Qian, K. Extremely Stretchable Strain Sensors Based on Conductive Self-Healing Dynamic Cross-Links Hydrogels for Human-Motion Detection. Adv. Sci. 2017, 4, 1600190. [CrossRef] [PubMed]

24. Tong, X.; Zheng, J.; Lu, Y. Swelling and mechanical behaviors of carbon nanotube/poly(vinyl alcohol) hybrid hydrogels. Mater. Lett. 2007, 61, 1704-1706. [CrossRef]

25. Hur, J.; Im, K.; Kim, S.W. Polypyrrole/agarose-based electronically conductive and reversibly restorable hydrogel. ACS Nano 2014, 8, 10066-10076. [CrossRef] [PubMed]

26. Shi, Y.; Wang, M.; Ma, C. A Conductive Self-Healing Hybrid Gel Enabled by Metal-Ligand Supramolecule and Nanostructured Conductive Polymer. Nano Lett. 2015, 15, 6276-6281. [CrossRef] [PubMed]

27. Wei, Z.; Yang, J.H.; Zhou, J. Self-healing gels based on constitutional dynamic chemistry and their potential applications. Chem. Soc. Rev. 2014, 43, 8114-8131. [CrossRef]

28. Huynh, T.; Haick, H. Self-Healing, Fully Functional, and Multiparametric Flexible Sensing Platform. Adv. Mater. 2016, 28, 138-143. [CrossRef] 
29. Han, J.; Zhou, C.; Wu, Y. Self-Assembling Behavior of Cellulose Nanoparticles during Freeze-Drying: Effect of Suspension Concentration, Particle Size, Crystal Structure, and Surface Charge. Biomacromolecules 2013, 14, 1529-1540. [CrossRef]

30. Han, J.; Zhou, C.; French, A.D. Characterization of cellulose II nanoparticles regenerated from 1-butyl-3-methylimidazolium chloride. Carbohydr. Polym. 2013, 94, 773-781. [CrossRef]

31. Cao, J.; Zhang, X.; Wu, X. Cellulose nanocrystals mediated assembly of graphene in rubber composites for chemical sensing applications. Carbohydr. Polym. 2016, 140, 88-95. [CrossRef] [PubMed]

32. Xu, S.; Yu, W.; Yao, X. Nanocellulose-assisted dispersion of graphene to fabricate poly(vinyl alcohol)/graphene nanocomposite for humidity sensing. Compos. Sci. Technol. 2016, 131, 67-76. [CrossRef]

33. Hajian, A.; Lindström, S.B.; Pettersson, T. Understanding the Dispersive Action of Nanocellulose for Carbon Nanomaterials. Nano Lett. 2017, 17, 1439-1447. [CrossRef] [PubMed]

34. Bai, L.; Bossa, N.; Qu, F. Comparison of Hydrophilicity and Mechanical Properties of Nanocomposite Membranes with Cellulose Nanocrystals and Carbon Nanotubes. Environ. Sci. Technol. 2017, 51, 253-262. [CrossRef] [PubMed]

35. Han, J.; Lu, K.; Yue, Y. Nanocellulose-templated assembly of polyaniline in natural rubber-based hybrid elastomers toward flexible electronic conductors. Ind. Crops Prod. 2019, 128, 94-107. [CrossRef]

36. Han, J.; Yue, Y.; Wu, Q. Effects of nanocellulose on the structure and properties of poly(vinyl alcohol)-borax hybrid foams. Cellulose 2017, 24, 4433-4448. [CrossRef]

37. Lu, B.; Lin, F.; Jiang, X. One-Pot Assembly of Microfibrillated Cellulose Reinforced PVA-Borax Hydrogels with Self-Healing and $\mathrm{pH}$-Responsive Properties. ACS Sustain. Chem. Eng. 2016, 5, 948-956. [CrossRef]

38. Ding, Q.; Xu, X.; Yue, Y. Nanocellulose-Mediated Electroconductive Self-Healing Hydrogels with High Strength, Plasticity, Viscoelasticity, Stretchability, and Biocompatibility toward Multifunctional Applications. ACS Appl. Mater. Interfaces 2018, 10, 27987-28002. [CrossRef]

39. Zhong, M.; Liu, Y.T.; Xie, X.M. Self-healable, super tough graphene oxide-poly (acrylic acid) nanocomposite hydrogels facilitated by dual cross-linking effects through dynamic ionic interactions. J. Mater. Chem. B 2015, 3, 4001-4008. [CrossRef]

40. Wang, Q.; Mynar, J.L.; Yoshida, M. High-water-content mouldable hydrogels by mixing clay and a dendritic molecular binder. Nature 2010, 463, 339-343. [CrossRef]

41. Han, J.; Lei, T.; Wu, Q. Facile preparation of mouldable polyvinyl alcohol-borax hydrogels reinforced by well-dispersed cellulose nanoparticles: Physical, viscoelastic and mechanical properties. Cellulose 2013, 20, 2947-2958. [CrossRef]

42. Wang, Y.; Chen, L. Impacts of nanowhisker on formation kinetics and properties of all-cellulose composite gels. Carbohydr. Polym. 2011, 83, 1937-1946. [CrossRef]

43. Zhou, C.; Wu, Q. A novel polyacrylamide nanocomposite hydrogel reinforced with natural chitosan nanofibers. Coll. Surf. B Biointerfaces 2011, 84, 155-162. [CrossRef] [PubMed]

44. Shao, C.; Chang, H.; Wang, M. High-Strength, Tough, and Self-Healing Nanocomposite Physical Hydrogels Based on the Synergistic Effects of Dynamic Hydrogen Bond and Dual Coordination Bonds. ACS Appl. Mater. Interfaces 2017, 9, 28305-28318. [CrossRef] [PubMed]

45. Yang, W.; Shao, B.; Liu, T. Robust and Mechanically and Electrically Self-Healing Hydrogel for Efficient Electromagnetic Interference Shielding. ACS Appl. Mater. Interfaces 2018, 10, 8245-8257. [CrossRef] [PubMed]

46. Liu, X.; Su, G.; Guo, Q. Hierarchically Structured Self-Healing Sensors with Tunable Positive/Negative Piezoresistivity. Adv. Funct. Mater. 2018, 28, 1706658. [CrossRef]

47. Gui, Z.; Zhu, H.; Gillette, E. Natural cellulose fiber as substrate for supercapacitor. ACS Nano 2013, 7, 6037-6046. [CrossRef]

48. Wang, Z.; Tammela, P.; Zhang, P. Freestanding nanocellulose-composite fibre reinforced 3D polypyrrole electrodes for energy storage applications. Nanoscale 2014, 6, 13068-13075. [CrossRef] [PubMed]

49. Liu, S.; Li, L. Ultrastretchable and self-healing double-network hydrogel for 3D printing and strain sensor. ACS Appl. Mater. Interfaces 2017, 9, 26429-26437. [CrossRef]

50. Jing, X.; Mi, H.Y.; Lin, Y.J. Highly Stretchable and Biocompatible Strain Sensors Based on Mussel-Inspired Super-Adhesive Self-Healing Hydrogels for Human Motion Monitoring. ACS Appl. Mater. Interfaces 2018, 10, 20897-20909. [CrossRef] 
51. Guo, Q.; Luo, Y.; Liu, J. A well-organized graphene nanostructure for versatile strain-sensing application constructed by a covalently bonded graphene/rubber interface. J. Mater. Chem. C 2018, 6, 2139-2147. [CrossRef]

52. Liu, X.; Lu, C.; Wu, X. Self-healing strain sensors based on nanostructured supramolecular conductive elastomers. J. Mater. Chem. A 2017, 5, 9824-9832. [CrossRef]

53. Guo, Q.; Cao, J.; Han, Y. Biological phytic acid as a multifunctional curing agent for elastomers: Towards skin-touchable and flame retardant electronic sensors. Green Chem. 2017, 19, 3418-3427. [CrossRef]

54. Wang, C.; Zhao, J.; Ma, C. Detection of non-joint areas tiny strain and anti-interference voice recognition by micro-cracked metal thin film. Nano Energy 2017, 34, 578-585. [CrossRef]

55. Cao, J.; Lu, C.; Zhuang, J. Multiple Hydrogen Bonding Enables the Self-Healing of Sensors for Human-Machine Interactions. Angew. Chem. Int. Ed. 2017, 56, 8795-8800. [CrossRef] [PubMed]

56. Wei, Y.; Chen, S.; Lin, Y. Cu-Ag core-shell nanowires for electronic skin with a petal molded microstructure. J. Mater. Chem. C 2015, 3, 9594-9602. [CrossRef]

57. Liu, S.; Zheng, R.; Chen, S. A compliant, self-adhesive and self-healing wearable hydrogel as epidermal strain sensor. J. Mater. Chem. C 2018, 6, 4183-4190. [CrossRef]

(C) 2019 by the authors. Licensee MDPI, Basel, Switzerland. This article is an open access article distributed under the terms and conditions of the Creative Commons Attribution (CC BY) license (http://creativecommons.org/licenses/by/4.0/). 